PROCEEDINGS OF THE

AMERICAN MATHEMATICAL SOCIETY

Volume 129, Number 11, Pages 3231-3239

S 0002-9939(01)05980-9

Article electronically published on April 16, 2001

\title{
A UNIVERSAL PROPERTY OF REFLEXIVE HEREDITARILY INDECOMPOSABLE BANACH SPACES
}

\author{
SPIROS A. ARGYROS
}

(Communicated by N. Tomczak-Jaegermann)

\begin{abstract}
It is shown that every separable Banach space $X$ universal for the class of reflexive Hereditarily Indecomposable space contains $C[0,1]$ isomorphically and hence it is universal for all separable spaces. This result shows the large variety of reflexive H.I. spaces.
\end{abstract}

\section{INTRODUCTION}

In 1980 J. Bourgain $[\mathrm{B}$ proved that every separable Banach space $X$ containing an isomorphic copy of any separable reflexive space must also contain isomorphically any separable Banach space. This theorem extended previous results proven by W. Szlenk [S]. Our goal, in this paper, is to show that the conclusion of Bourgain's theorem remains valid if we assume that $X$ contains a subclass of separable reflexive spaces, namely the class of reflexive Hereditarily Indecomposable (H.I.) spaces. We recall that a Banach space $X$ is H.I. if every infinite dimensional closed subspace $Y$ of $X$ has no non-trivial decomposition into a direct sum of two Banach spaces. A classical result, due to J. Lindenstrauss $[\mathrm{L}$, states that every non-separable reflexive Banach space $X$ contains a complemented separable subspace. Hence the class of reflexive H.I. Banach spaces contains only separable spaces. The concept of an H.I. space followed the construction of the celebrated W. Gowers - B. Maurey example of a Banach space with no unconditional basic sequence [G-M]. Since then the class of H.I. spaces has been studied by several researchers. We refer the reader to $[\mathrm{A}-\mathrm{F}$ ] where the development of the theory is explained. In the same paper the following dichotomy is proved: Every Banach space $X$ either contains an isomorphic copy of $\ell^{1}$ or else it has an infinite dimensional closed subspace which is a quotient of an H.I. Banach space. This theorem yields that separable H.I. Banach spaces are not just certain scattered examples but define a large class of Banach spaces. The result that we obtain in this paper is another strong evidence of this assertion.

Our proof depends on Bourgain's techniques of constructing reflexive Banach spaces connected to well founded trees and also it makes use of results contained in $\mathrm{A}-\mathrm{F}$. The paper is organized into two sections. The first is devoted to recalling definitions and results contained in $[\mathrm{B}$, with some small modifications which are

Received by the editors March 5, 2000.

2000 Mathematics Subject Classification. Primary 46B03, 46B70, 46B10; Secondary 03E10, $03 \mathrm{E} 15$.

Key words and phrases. Reflexive Banach spaces, hereditarily indecomposable Banach spaces, universal Banach spaces, well-founded trees. 
neccessary for our proofs. The second section contains results from $\mathrm{A}-\mathrm{F}$, as well as the proof of the theorem mentioned in the abstract. The precise statement is as follows.

Theorem 1.1. Every separable Banach space $X$ containing an isomorphic copy of any reflexive H.I. Banach space also contains an isomorphic copy of any separable Banach space.

\section{BOURGAIN'S CLASS $\left\{R_{\xi}(Y)\right\}_{\xi<\omega_{1}}$}

This section is devoted to the construction of a family of Banach spaces $\left(T_{\xi}\right)_{\xi<\omega_{1}}$ of well-founded trees and a family $\left(R_{\xi}(Y)\right)_{\xi<\omega_{1}}$ where $Y$ is a Banach space with a Schauder basis. Our approach closely follows J. Bourgain's ideas [B].

2.1. Well-founded trees. We begin by recalling certain definitions concerning the well-founded trees.

Definition 2.1. (i) A tree $T$ is any partially ordered set, such that for every $t \in T$ the set $\{s \in T: s<t\}$ is well ordered. A tree is called well-founded provided there is no strictly increasing sequence $\left\{t_{n}\right\}_{n \in \mathbb{N}}$ of elements of $T$.

(ii) An element $t$ of a well-founded tree $T$ is called maximal if for every $s \in T$ with $t \leq s$ we have that $t=s$.

(iii) For a well-founded tree $T$ we define $T^{\prime}=T \backslash\{t \in T: t$ is maximal $\}$ and then we inductively define

$$
T^{(0)}=T, T^{(\xi+1)}=\left(T^{(\xi)}\right)^{\prime}, T^{(\xi)}=\bigcap_{\zeta<\xi} T^{(\zeta)}
$$

where the last definition concerns limit ordinals $\xi$.

(iv) The order $o(T)$ of a well-founded tree is defined as:

$$
o(T)=\min \left\{\xi: T^{(\xi)}=\emptyset\right\} .
$$

It is clear that for every well-founded tree $T$ the order $o(T)$ is well defined and further if $T$ is a countable set, then $o(T)$ is a countable ordinal.

(v) In the sequel for $t \in T$ we set $|t|=\#\{s \in T: s<t\}$ which, in the case of a well-founded tree $T$, is always a natural number. Further a segment of $T$ is a subset of the form $S=\left\{t: s_{1} \leq t \leq s_{2}\right\}$. An initial segment is a segment for which $s_{1}$ is a minimal element of $T$.

Next we define a family $\left(T_{\xi}\right)_{\xi<\omega_{1}}$ of well-founded trees, such that each $T_{\xi}$ is a countable set and $o\left(T_{\xi}\right)=\xi$ for every $\xi<\omega_{1}$. The existence of such a family is well known but we recall its definition since we shall use certain of its properties.

We set $T_{0}=\emptyset$. If $T_{\xi}$ has been defined we set $T_{\xi+1}=\left\{t_{\xi+1}\right\} \cup T_{\xi}$ where $t_{\xi+1}$ denotes a new object not belonging to $T_{\xi}$. We define an order on $T_{\xi+1}$ as follows. For every $t, s \in T_{\xi} t<_{\xi+1} s$ iff $t<_{\xi} s$ while if $t \in T_{\xi}$ we set $t_{\xi+1}<_{\xi+1} t$. It is easy to check that $T_{\xi+1}$ is a tree and $o\left(T_{\xi+1}\right)=o\left(T_{\xi}\right)+1=\xi+1$. If $\xi$ is a limit ordinal and $\left\{T_{\zeta}\right\}_{\zeta<\xi}$ have been defined we choose a strictly increasing sequence $\left(\zeta_{n}\right)_{n \in \mathbb{N}}$ such that $\lim \zeta_{n}=\xi$. We may also assume that $\left(T_{\zeta_{n}}\right)_{n \in \mathbb{N}}$ are pairwise disjoint and we define $T_{\xi}=\bigcup_{n=1}^{\infty} T_{\zeta_{n}}$ ordered in the natural manner. It is also easy to see that $T_{\xi}$ is well-founded and $o\left(T_{\xi}\right)=\xi$. The following properties are straightforward consequences of the inductive definition. We state them explicitely for later use.

The family $\left(T_{\xi}\right)_{\xi<\omega_{1}}$ satisfies the following:

(i) Each $T_{\xi}$ is a countable set. 
(ii) If $\xi=\zeta+1$, then $T_{\xi}=\left\{t_{\xi}\right\} \cup T_{\zeta}$.

(iii) If $\xi$ is a limit ordinal, then $T_{\xi}=\bigcup_{n=1}^{\infty} Q_{n}$ such that $\left\{Q_{n}\right\}_{n \in \mathbb{N}}$ are pairwise incomparable subtrees (i.e. for $n_{1} \neq n_{2}, t_{1} \in Q_{n_{1}}, t_{2} \in Q_{n_{2}}$ then $t_{1}, t_{2}$ are incomparable) and $o\left(Q_{n}\right)<\xi$.

2.2. Tree representation of Banach spaces. Let $X$ be a Banach space. Following J. Bourgain [B] we consider the tree $T_{X}=\bigcup_{n=1}^{\infty} X^{n}$ ordered by the relation $\left(y_{1}, \ldots, y_{m}\right)<\left(x_{1}, \ldots, x_{n}\right)$ iff $m<n$ and for all $1 \leq i \leq m, y_{i}=x_{i}$. Next for $X, Y$ Banach spaces, $\left(y_{n}\right)_{n}$ a Schauder basis of $Y$ and $\varepsilon>0$, we define

$$
\begin{aligned}
& T\left(X, Y,\left(y_{n}\right), \varepsilon\right) \\
& \quad=\left\{\left(x_{1}, \ldots, x_{n}\right) \in T_{X}: \varepsilon\left\|\sum_{k=1}^{n} a_{k} y_{k}\right\| \leq\left\|\sum_{k=1}^{n} a_{k} x_{k}\right\| \leq \varepsilon^{-1}\left\|\sum_{k=1}^{n} a_{k} y_{k}\right\|\right\},
\end{aligned}
$$

where the inequalities hold for all $\left(a_{i}\right)_{i=1}^{n} \in \mathbb{R}^{n}$.

It follows that $T\left(X, Y,\left(y_{n}\right), \varepsilon\right)$ is a subtree of $T_{X}$ and further on, the space $Y$ is not isomorphic to a subspace of $X$ iff for all $\varepsilon>0$ the corresponding tree $T\left(X, Y,\left(y_{n}\right), \varepsilon\right)$ is well-founded. Moreover, J. Bourgain has proved that:

Theorem 2.2. Let $X, Y$ be separable Banach spaces such that $Y$ has a Schauder basis $\left(y_{n}\right)_{n}$ and $X$ contains no isomorphic copy of $Y$. Then for every $\varepsilon>0$

$$
o\left(T\left(X, Y,\left(y_{n}\right)_{n}, \varepsilon\right)\right)<\omega_{1} .
$$

As a consequence of this theorem we have the following corollary.

Corollary 2.3. Let $Y$ be a separable Banach space with a Schauder basis $\left(y_{n}\right)_{n}$. Then $Y$ is isomorphic to a subspace of a separable space $X$ if and only if for all $\xi<\omega_{1}$ there exist $\varepsilon>0$ and a subtree $S_{\xi} \subset T\left(X, Y,\left(y_{n}\right)_{n}, \varepsilon\right)$ with $S_{\xi}$ well-founded and $o\left(S_{\xi}\right) \geq \xi$.

The family $\left\{R_{\xi}(Y)\right\}_{\xi<\omega_{1}}$. For the remaining of the paper $Y$ will denote a separable Banach space with a fixed Schauder basis $\left(y_{n}\right)_{n}$. We also recall that for a non-empty set $T, c_{00}(T)$ denotes the vector space of all finitely supported real valued functions defined on $T$. For $t \in T$ we denote by $e_{t}$ the characteristic function of $\{t\}$. For an infinite countable ordinal $\xi$ we define the following norm on $c_{00}\left(T_{\xi}\right)$. ( $T_{\xi}$ denotes the well-founded tree defined above.) For $x \in c_{00}\left(T_{\xi}\right)$ we set

$$
\begin{array}{r}
\|x\|_{\xi}=\sup \left\{\left(\sum_{i=1}^{\ell}\left\|\sum_{t \in S_{i}} x(t) \cdot y_{|t|+1}\right\|^{2}\right)^{1 / 2}:\right. \\
\left.\left\{S_{i}\right\}_{i=1}^{\ell} \text { are pairwise incomparable segments of } T_{\xi}\right\} .
\end{array}
$$

Finally we denote by $R_{\xi}(Y)$ the completion of $\left(c_{00}\left(T_{\xi}\right),\|\cdot\|_{\xi}\right)$.

Proposition 2.4. For every $\omega \leq \xi<\omega_{1}$ and every $Y$ the following hold:

(i) $R_{\xi}(Y)$ is a reflexive space.

(ii) There exists $\varepsilon>0$ such that $T_{\xi}$ is embedded as a subtree in $T\left(R_{\xi}(Y), Y\right.$, $\left.\left(y_{n}\right)_{n}, \varepsilon\right)$. 
Proof. (i) We make the following inductive assertion:

For every $\omega \leq \xi<\omega_{1}$ and every $Y$ the space $R_{\xi}(Y)$ is reflexive. This will be shown by induction.

We begin with $\xi=\omega$. From the definition of $T_{\omega}$ we have that $T_{\omega}=\bigcup_{n=1}^{\infty} T_{n}$ such that each $T_{n}$ is a well-ordered finite set. Now for any $Y$ with a Schauder basis $\left(y_{n}\right)_{n \in \mathbb{N}}$ we observe that

$$
R_{\omega}(Y)=\left(\sum_{n=1}^{\infty} \oplus R_{n}(Y)\right)_{2} .
$$

In the above each $R_{n}(Y)$ is a finite dimensional space isomorphic, with constant $M>0$, independent to $n$, to a subspace of $Y$ generated by $\left\{y_{1}, \ldots, y_{k_{n}}\right\}$ where $k_{n}=\operatorname{dim} R_{n}$. Hence $R_{\omega}(Y)$ is a reflexive space. To treat the general case assume that for all $\zeta<\xi$ and all spaces $Y$ the space $R_{\zeta}(Y)$ is reflexive. We first consider the case of a successor ordinal $\xi=\zeta+1$.

In this case $R_{\xi}(Y) \cong \mathbb{R} \oplus R_{\zeta}\left(Y^{\prime}\right)$ where $Y^{\prime}=\overline{\left\langle\left\{y_{n}\right\}_{n \geq 2}\right\rangle}$ and by the inductive assumption we have that $R_{\zeta}\left(Y^{\prime}\right)$ is reflexive. This yields the reflexivity of $R_{\xi}(Y)$. Next if $\xi$ is a limit ordinal and $\left(\zeta_{n}\right)_{n}$ is the sequence of ordinals used in the definition of $T_{\xi}$, then we easily conclude that

$$
R_{\xi}(Y)=\left(\sum_{n=1}^{\infty} \oplus R_{\zeta_{n}}(Y)\right)_{2}
$$

and by the inductive assumption each $R_{\zeta_{n}}(Y)$ is reflexive. Thus $R_{\xi}(Y)$ is also reflexive and this completes the proof for (i).

(ii) Consider the set

$$
S_{\xi}^{\prime}=\left\{\left(e_{t_{1}}, e_{t_{2}}, \ldots, e_{t_{n}}\right):\left\{t_{i}\right\}_{i=1}^{n} \text { is an initial segment of } T_{\xi}\right\} .
$$

Then clearly $S_{\xi}^{\prime}$ defines a subtree of $T_{R_{\xi}(Y)}$ isomorphic to $T_{\xi}$. Moreover every $\left(e_{t_{i}}, \ldots, e_{t_{n}}\right)$ in $S_{\xi}^{\prime}$ is $M$-equivalent to $\left(y_{1}, \ldots, y_{n}\right)$, the initial segment of the basis $\left(y_{n}\right)_{n}$ of the space $Y$, with $M$ independent to $n$. This shows that for $\varepsilon=\frac{1}{M}$ the tree $S_{\xi}^{\prime}$ is a subtree of $T\left(R_{\xi}(Y), Y, \varepsilon\right)$ and this completes the proof.

\section{Hereditarily indeComposable SpaCes}

We start by recalling, from $\mathrm{A}-\mathrm{F}$, the definitions of thin and a-thin sets.

Definition 3.1. Let $W$ be a convex, symmetric, bounded and closed subset of a Banach space $X$. Then:

(a) The set $W$ is said to be thin if for every $Y$ infinite dimensional subspace of $X$ there exists $\varepsilon>0$ such that for all $\lambda \in \mathbb{R}, B_{Y} \not \subset \lambda W+\varepsilon B_{X}$.

(b) The set $W$ is a-thin where $\mathbf{a}=\left(a_{n}\right)$ is a null sequence of positive real numbers provided the equivalent norms $\left\{\|\cdot\|_{n}\right\}_{n}$ defined by Minkowski's gauges $\left\{2^{n} W+\right.$ $\left.a_{n} B_{X}\right\}_{n}$ are not uniformly bounded on $B_{Y}$ for every infinite dimensional subspace $Y$ of the space $X$.

The concepts of thin and a-thin sets play a key role in the study of quotients of H.I. Banach spaces. Thin sets were introduced in $[\mathbb{N}]$ while the notion of a-thin sets is due to B. Maurey $([\mathrm{A}-\mathrm{F}])$. It follows readily from the definition that every thin set is also a-thin for every null sequence $\mathrm{a}=\left(a_{n}\right)_{n}$. Further if $W$ is a-thin, then it is also b-thin for every sequence $b=\left(b_{n}\right)_{n}$ with $0<b_{n} \leq a_{n}$. 
Next we briefly recall how the a-thin property of a set $W$ is used in $\mathrm{A}-\mathrm{F}$.

Suppose that $W$ is an a-thin set in a separable Banach space $X$. We denote by $X_{n}$ the space $\left(X,\|\cdot\|_{n}\right)$ where $\|\cdot\|_{n}$ denotes the equivalent norm defined by the gauge $2^{n} \cdot W+a_{n} B_{X}$. Consider the vector space $\left(\sum_{n=1}^{\infty} \oplus X_{n}\right)_{00}$ of all sequences $\left(x_{n}\right)_{n \in \mathbb{N}}$ which are eventually zero. One of the main results in $[\mathrm{A}-\mathrm{F}$ is the following.

Theorem 3.2. There exists a norm defined on $\left(\sum_{n=1}^{\infty} \oplus X_{n}\right)_{00}$ such that if we denote by $\widetilde{X}=\left(\sum_{n=1}^{\infty} \oplus X_{n}\right)_{*}$ its completion, then the following hold:

(i) The sequence $\left(X_{n}\right)_{n}$ defines a Schauder decomposition of $\widetilde{X}$ (i.e. every $\widetilde{x} \in \widetilde{X}$ has a unique representation of the form $\sum_{n=1}^{\infty} x_{n}$, with $x_{n} \in X_{n}$ ).

(ii) If we denote by $\Delta \widetilde{X}$ the closed subspace of $\widetilde{X}$ consisting of all elements of the form $(x, x, \ldots, x, \ldots), x \in X$, with finite norm, then $\Delta \widetilde{X}$ is an H.I. space.

(iii) Moreover if the initial space $X$ is reflexive, then the same holds for the space $\widetilde{X}$ and hence $\Delta \widetilde{X}$ is also reflexive.

Let us observe that for $x \in W$ we have that $\|(x, x, \ldots)\|_{*} \leq 1$ and therefore it belongs to $\Delta \widetilde{X}$. Furthermore the projection $j: \Delta \widetilde{X} \rightarrow X_{1}$ is a bounded linear injection. Hence the above theorem yields the next.

Theorem 3.3. Let $X$ be a separable Banach space and let $W$ be an $\mathbf{a}$-thin subset of $X$. Then there exists an H.I. space $\Delta$ and a bounded linear one-to-one operator $j: \Delta \rightarrow X$ such that $j\left(B_{\Delta}\right)$ contains the set $W$. If $X$ is reflexive, then $\Delta$ can also be chosen to be reflexive. Moreover, if $F$ is a finite dimensional subspace of $X$ such that $B_{F} \subset W$, then $j^{-1}: F \rightarrow \Delta$ defines an isomorphism with $\left\|\left.j^{-1}\right|_{F}\right\| \cdot\left\|\left.j\right|_{F}\right\| \leq M$ with $M$ independent of $F$.

We now return to the family $\left(R_{\xi}(Y)\right)_{\omega \leq \xi<\omega_{1}}$ with the following.

Definition 3.4. Let $\omega \leq \xi<\omega_{1}$ be an ordinal and let $Y$ be a separable Banach space with a Schauder basis $\left(y_{n}\right)_{n}$. In the space $R_{\xi}(Y)$, defined in the previous section, we define a set $W_{\xi}(Y)$ as follows. First we consider the set $\mathcal{M}_{\xi}=\{S \subset$ $T_{\xi}: S$ is a maximal initial segment of $\left.T_{\xi}\right\}$. Given $S \in \mathcal{M}_{\xi}$ we denote by $B_{S}$ the unit ball of the (finite dimensional) subspace generated by the set $\left\{e_{t}: t \in S\right\}$. Finally we set

$$
W_{\xi}(Y)=\overline{c o}\left(\bigcup\left\{B_{S}: S \in \mathcal{M}_{\xi}\right\}\right)
$$

Observe that $W_{\xi}(Y)$ is a closed, bounded, symmetric, convex subset.

The following will be proved.

Proposition 3.5. For all $\omega \leq \xi<\omega_{1}$ and every separable space $Y$ with a basis $\left(y_{n}\right)_{n}$ the set $W_{\xi}(Y)$ is a thin subset of $R_{\xi}(Y)$.

Let us point out that Proposition 3.5 in conjunction with Proposition 2.4 and Theorem 3.3 yield

Theorem 3.6. For every $\omega \leq \xi<\omega_{1}$ and every separable space $Y$ with a basis $\left(y_{n}\right)_{n}$ there exists a Banach space $H_{\xi}(Y)$ satisfying the following:

(a) $H_{\xi}(Y)$ is a reflexive H.I. space.

(b) There exists $\varepsilon>0$ and

$$
S_{\xi} \subset T\left(H_{\xi}(Y), Y,\left(y_{n}\right)_{n}, \varepsilon\right)
$$

such that $S_{\xi}$ is a well-founded subtree of $T\left(H_{\xi}(Y), Y,\left(y_{n}\right), \varepsilon\right)$ and $o\left(S_{\xi}\right) \geq \xi$. 
Proof of Theorem 1.1. Observe that applying Theorem 3.6 for the space $Y=C[0,1]$ and any Schauder basis $\left(y_{n}\right)_{n}$ of $Y$, we obtain a family $\left\{H_{\xi}(Y)\right\}_{\omega \leq \xi<\omega_{1}}$ of reflexive H.I. spaces such that if a separable Banach space $X$ contains an isomorphic copy of each $H_{\xi}(Y)$, then Bourgain's theorem (Theorem 2.2) enforces the embedding of $C[0,1]$ into $X$. Thus $X$ is universal for the class of separable Banach space. This completes the proof of Theorem 1.1.

Hence it remains to show how we obtain Proposition 3.5. We start with the following known Ramsey type result.

Lemma 3.7. Let $X,\left\{X_{i}\right\}_{i=1}^{n}$ be Banach spaces and assume that there exists an isomorphic embedding $T: X \rightarrow \sum_{i=1}^{n} \oplus X_{i}$. Then there exists an infinite dimensional subspace $Y$ of $X$ and $1 \leq i \leq n$ such that $P_{i} \circ T: Y \rightarrow X_{i}$ is an isomorphism, where $P_{i}$ is the natural projection onto $X_{i}$.

Proof. Observe that it is enough to prove the statement for $n=2$. In this case, assuming that $P_{1} \circ T$ is a strictly singular operator we obtain a normalized sequence $\left(y_{n}\right)_{n \in \mathbb{N}}$ in $X$ which is Schauder basic and such that

$$
\sum_{n=1}^{\infty}\left\|T\left(y_{n}\right)-\left(0, P_{2} \circ T\left(y_{n}\right)\right)\right\| \leq 1 .
$$

Then by standard arguments (cf. [L-T] ) we obtain a subsequence $\left(y_{n}\right)_{n \in M}$ such that $P_{2} \circ T: Y \rightarrow X_{2}$ is an isomorphism. In the above $Y$ denotes the space $\overline{\left\langle\left\{y_{n}\right\}_{n \in M}\right\rangle}$.

Before passing to the proof of Proposition 3.5 we make some preparatory observations concerning the structure of $R_{\xi}(Y)$. In the sequel, two finitely supported elements $z_{1}, z_{2}$ of $R_{\xi}(Y)$ (i.e. $\left.z_{i} \in c_{00}\left(T_{\xi}\right), i=1,2\right)$ will be called incomparable iff for every $t_{i} \in \operatorname{supp} z_{i}, i=1,2$, we have $t_{1}$ is incomparable to $t_{2}$. A similar definition holds for $R_{\xi}^{*}(Y)$.

Lemma 3.8. Let $\left(z_{n}\right)_{n \in \mathbb{N}}$ be a seminormalized sequence of pairwise incomparable elements of $R_{\xi}(Y)$. Then there exists a sequence $\left(z_{n}^{*}\right)_{n \in \mathbb{N}}$ in $R_{\xi}^{*}(Y)$ such that the following are fulfilled:

(i) Each $z_{n}^{*}$ has finite support. This means that each $z_{n}^{*}$ is a linear combination of $\left\{e_{t}^{*}: t \in T_{\xi}\right\}$.

(ii) The sequence $\left(z_{n}^{*}\right)_{n \in \mathbb{N}}$ consists of pairwise incomparable elements.

(iii) $\left\|z_{n}^{*}\right\| \leq 1$ and $z_{n}^{*}\left(z_{n}\right)>\frac{\left\|z_{n}\right\|}{2}$.

Proof. It follows from the definition of $\|\cdot\|_{\xi}$ on $R_{\xi}(Y)$ that if $\left\{S_{i}\right\}_{i=1}^{\ell}$ are incomparable segments such that

$$
\frac{\left\|z_{n}\right\|}{2}<\left(\sum_{i=1}^{\ell}\left\|\sum_{t \in S_{i}} z_{n}(t) \cdot y_{|t|+1}\right\|^{2}\right)^{1 / 2},
$$

then there exists $z_{n}^{*}$ supported by $\bigcup_{i=1}^{\ell}\left\{e_{t}^{*}: t \in S_{i}\right\}$ with $\left\|z_{n}^{*}\right\| \leq 1$ and $z_{n}^{*}\left(z_{n}\right)>$ $\frac{\left\|z_{n}\right\|}{2}$. Also we can assume that the collection $\left\{S_{i}\right\}_{i=1}^{\ell}$ satisfies the following:

For each $i=1,2, \ldots, \ell$ there exist $t(i, 1), t(i, 2)$ in supp $z_{n}$ such that

$$
t(i, 1) \leq \min S_{i} \leq \max S_{i} \leq t(i, 2) .
$$

The last condition yields that if $\left(z_{n}\right)_{n \in \mathbb{N}}$ are pairwise incomparable, then the same holds for the sequence $\left(z_{n}^{*}\right)_{n \in \mathbb{N}}$ and this completes the proof. 
Remark 3.9. A direct consequence of the definition of the norm of $R_{\xi}(Y)$ is that for every bounded, seminormalised sequence $\left(z_{n}\right)_{n \in \mathbb{N}}$ of pairwise incomparable elements of $R_{\xi}(Y)$ the following hold:

(i) The space $Z=\overline{\left\langle\left(z_{n}\right)_{n}\right\rangle}$ is isometric to $\ell^{2}$.

(ii) If $\left(z_{n}^{*}\right)_{n \in \mathbb{N}}$ denotes a sequence in the dual $R_{\xi}^{*}(Y)$ such that $\left(z_{n}^{*}\right)_{n \in \mathbb{N}}$ are pairwise incomparable, $\left\|z_{n}^{*}\right\| \leq 1$ and $z_{n}^{*}\left(z_{n}\right)>\frac{\left\|z_{n}\right\|}{2}>\vartheta>0$, then the map $P(x)=$ $\sum_{n=1}^{\infty} \frac{z_{n}^{*}(x) \cdot z_{n}}{z_{n}^{*}\left(z_{n}\right)}$ defines a bounded projection

$$
P: R_{\xi}(Y) \rightarrow Z
$$

(iii) Furthermore for for every $S \in \mathcal{M}_{\xi}$ and $x \in B_{S}$ (Definition 3.4) we have $P(x)=\lambda \cdot z_{n}$ for some $n \in \mathbb{N}, \lambda \in \mathbb{R}$ and $|\lambda| \leq \vartheta^{-1}$.

Proof of Proposition 3.5. First we prove the following:

Claim 1. For every $\omega \leq \xi<\omega_{1}$ and every infinite dimensional closed subspace $B$ of $R_{\xi}(Y)$ there exists a seminormilized bounded sequence $\left(z_{n}\right)_{n \in \mathbb{N}}$ of pairwise incomparable elements of $R_{\xi}(Y)$ such that if $P: R_{\xi}(Y) \rightarrow Z=\overline{\left\langle\left(z_{n}\right)_{n}\right\rangle}$ denotes the projection defined above, then there exists a closed subspace $C$ of $B$ such that $\left.P\right|_{C}$ is an isomorphism between the spaces $C$ and $Z$.

To prove the claim we proceed by induction.

Case 1. $\xi=\omega$.

Observe the following:

(i) $R_{\omega}(Y)=\left(\sum_{n} \oplus R_{n}(Y)\right)_{2}$ with each $R_{n}(Y)$ being of finite dimension.

(ii) For every $z_{1}, z_{2}$ finitely supported vectors of $R_{\omega}(Y)$ we denote by

$$
A_{i}=\left\{n \in \mathbb{N}: \pi_{n}\left(z_{i}\right) \neq 0\right\}, i=1,2,
$$

where $\pi_{n}: R_{\omega}(Y) \rightarrow R_{n}(Y)$ are the natural projections. If the sets $A_{1}, A_{2}$ are disjoint, then $z_{1}, z_{2}$ are incomparable.

Now consider $B$ an infinite dimensional subspace of $R_{\omega}(Y)$. Since each $R_{n}(Y)$ is a finite dimensional space, using a sliding hump argument we obtain a sequence $\left(v_{k}\right)_{k \in \mathbb{N}} \subset B$ and a sequence $\left(z_{k}\right)_{k \in \mathbb{N}}$ in $R_{\omega}(Y)$ with the properties:

(a)

$$
\left\|v_{k}\right\|=1, \sum_{k=1}^{\infty}\left\|v_{k}-z_{k}\right\|<\frac{1}{8} .
$$

(b) If $A_{k}=\left\{n: \pi_{n}\left(z_{k}\right) \neq 0\right\}$, then $\left(A_{k}\right)_{k \in \mathbb{N}}$ consists of successive disjoint finite subsets of $\mathbb{N}$. Observation (ii), above, yields that $\left(z_{k}\right)_{k \in \mathbb{N}}$ consists of pairwise incomparable elements of $R_{\omega}(Y)$.

Now consider the projection $P: R_{\omega}(Y) \rightarrow Z=\overline{\left\langle\left(z_{n}\right)_{n}\right\rangle}$ defined in Remark 3.9 (ii). By standard arguments (cf. $[\overline{\mathrm{L}-\mathrm{T}}]$ ) one can show that $\left.P\right|_{\overline{\left\langle\left(v_{k}\right)_{k}\right\rangle}}$ defines an isomorphism between $C=\overline{\left\langle\left(v_{k}\right)_{k}\right\rangle}$ and $Z$.

This completes the proof for the case $\xi=\omega$.

Case 2. $\xi=\zeta+1$.

We may assume that the claim holds for $R_{\zeta}(Y)$, for any space $Y$.

Let $B$ be a closed infinite dimensional subspace of $R_{\xi}(Y)$. As we have shown in Section 2

$$
R_{\xi}(Y) \cong \mathbb{R} \oplus R_{\zeta}\left(Y^{\prime}\right)
$$


where $Y^{\prime}=\overline{\left\langle\left(y_{n}\right)_{n \geq 2}\right\rangle}$. Then clearly there exists a closed subspace $B_{1}$ of $B$ such that the natural projection $\pi: R_{\xi}(Y) \rightarrow R_{\zeta}\left(Y^{\prime}\right)$ defines an isomorphism on $B_{1}$. Now the result follows from the inductive assumption.

Case 3. $\xi$ is a limit ordinal.

For this case we need to work a little more. First we recall that $R_{\xi}(Y)=$ $\left(\sum_{n=1}^{\infty} \oplus R_{\xi_{n}}(Y)\right)_{2}$. Denote by $P_{n}$ the natural projection

$$
P_{n}: R_{\xi}(Y) \rightarrow\left(\sum_{\ell=1}^{n} \oplus R_{\xi_{\ell}}(Y)\right)_{2}
$$

For a given subspace $B$ of $R_{\xi}(Y)$ we consider the following subcases.

Subcase 3.1. There exist $n_{0} \in \mathbb{N}$ and a subspace $B_{1}$ of $B$ such that

$$
P_{n_{0} \mid B_{1}}: B_{1} \rightarrow\left(\sum_{\ell \leq n_{0}} \oplus R_{\xi_{\ell}}(Y)\right)_{2}
$$

is an isomorphism.

Then Lemma 3.7 yields the existence of a further subspace $B_{2}$ of $B_{1}$ and $1 \leq$ $\ell_{0} \leq n_{0}$ so that $\pi_{\ell_{0}}: B_{2} \rightarrow R_{\xi_{\ell_{0}}}(Y)$ is an into isomorphism. The desired result follows from our inductive assumption.

Subcase 3.2. Assume that for all $n \in \mathbb{N}, P_{n}: B \rightarrow\left(\sum_{\ell \leq n} \oplus R_{\xi_{\ell}}(Y)\right)_{2}$ is a strictly singular operator.

This is, of course, the negation of Subcase 3.1 and it means that for every subspace $B_{1}$ of $B$, every $n \in \mathbb{N}$ and $\varepsilon>0$ there exists $v \in B_{1}$ with $\|v\|=1$ and $\left\|P_{n}(v)\right\|<\varepsilon$. This permits us to apply a sliding hump argument to obtain two sequences $\left(v_{k}\right)_{k \in \mathbb{N}},\left(z_{k}\right)_{k \in \mathbb{N}}$ such that:

(a) $\left(v_{k}\right)_{k} \subset B,\left\|v_{k}\right\|=1, \sum_{k=1}^{\infty}\left\|v_{k}-z_{k}\right\|<\frac{1}{8}$.

(b) Each $z_{k}$ is finitely supported and if $A_{k}=\left\{n \in \mathbb{N}: \pi_{n}\left(z_{k}\right) \neq 0\right\}$, then $\left\{A_{k}\right\}_{k=1}^{\infty}$ consists of successive disjoint subsets of $\mathbb{N}$.

From (b) we conclude that $\left(z_{k}\right)_{k \in \mathbb{N}}$ are pairwise incomparable. Hence if we consider the projection $P: R_{\xi}(Y) \rightarrow Z=\overline{\left\langle\left(z_{k}\right)_{k}\right\rangle}$ defined in Remark 3.9 (ii) then from (a) we also obtain that $\left.P\right|_{C}$ is an isomorphism between $C$ and $Z$.

In the above $C$ denotes $\overline{\left\langle\left(v_{k}\right)_{k}\right\rangle}$. This completes the proof of Claim 1 .

We pass now to the last step of the proof by showing.

Claim 2. For all $\omega \leq \xi<\omega_{1}$ the set $W_{\xi}(Y)$ is a thin subset of $R_{\xi}(Y)$.

Proof of Claim 2. Assume on the contrary that there exist $\omega \leq \xi<\omega_{1}$ and a separable Banach space $Y$ such that $W=W_{\xi}(Y)$ is not a thin subset of $R_{\xi}(Y)$. Therefore there exists a subspace $C$ of $R_{\xi}(Y)$ such that for every $\varepsilon>0$ we can find $\lambda_{\varepsilon} \in \mathbb{R}$ with $B_{C} \subset \lambda_{\varepsilon} W+\varepsilon \cdot B_{R_{\xi}(Y)}$.

Claim 1 yields a sequence $\left(z_{k}\right)_{k \in \mathbb{N}}$ of pairwise incomparable elements of $R_{\xi}(Y)$ and a closed subspace $D$ of $C$ such that if $P: R_{\xi}(Y) \rightarrow Z$ is the projection defined in Remark 3.9, then $\left.P\right|_{D}: D \rightarrow Z$ is an onto isomorphism. Choose $\lambda \in \mathbb{R}$ such that $\lambda \cdot P\left(B_{D}\right)$ contains $B_{Z}$ and then choose $\varepsilon>0$ such that

$$
\varepsilon \cdot \lambda \cdot P\left(B_{R_{\xi}(Y)}\right) \subset \frac{1}{2} B_{Z} .
$$

Next choose $\lambda_{\varepsilon} \in \mathbb{R}$ such that

$$
B_{D} \subset \lambda_{\varepsilon} W+\varepsilon B_{R_{\xi}(Y)} .
$$


Hence (3.1) and (3.2) yield that

$$
B_{Z} \subset P\left(\lambda B_{D}\right) \subset \lambda \cdot \lambda_{\varepsilon} P(W)+\varepsilon \cdot \lambda \cdot P\left(B_{R_{\xi}(Y)}\right) \subset \lambda \cdot \lambda_{\varepsilon} P(W)+\frac{1}{2} B_{Z} .
$$

Therefore, by well known arguments (see also $[\mathrm{A}-\mathrm{F}]$ ) we obtain that $B_{Z} \subset 2 \cdot \lambda \cdot \lambda_{\varepsilon}$. $P(W)$.

Now, Remark 3.9 (iii) implies that there exists $\vartheta>0$ such that $P(W) \subset$ $\overline{c o}\left\{ \pm \vartheta^{-1} z_{n}\right\}_{n}$. Therefore $B_{Z} \subset \overline{c o}\left\{ \pm \vartheta^{-1} z_{n}\right\}$ which of course derives a contradiction since otherwise the $\ell^{2}$-norm would be equivalent to the $\ell^{1}$-norm.

This completes the entire proof of the proposition.

We would like to thank Ioannis Gasparis and the referee for their comments on the content of the present paper.

\section{REFERENCES}

[A-F] S.A. Argyros and V. Felouzis. Interpolating Hereditarily Indecomposable Banach spaces, Journal of AMS 13 (2000) 243-294. CMP 2000:11

[B] J. Bourgain. On separable Banach spaces universal for all separable reflexive spaces, Proc. of AMS 79(1980) 241-246. MR 81f:46021

[G-M] W.T. Gowers and B. Maurey. The unconditional basic sequence problem, Journal of AMS 6(1993) 851-874. MR 94k:46021

[L-T] J. Lindenstrauss and L. Tzafriri. Classical Banach spaces I, Spinger Verlag 92(1977). MR 58:17766

[L] J. Lindenstrauss. On non-separable reflexive Banach spaces, Bulletin AMS 72(1996), 967970.

[N] R. Neidinger. Properties of Tauberian Operators, Dissertation. University of Texas at Austin, 1984

[S] W. Szlenk. The non-existence of a separable reflexive Banach spaces universal for all separable reflexive Banach spaces, Studia Math. 30(1968) 53-61. MR 37:3327

Department of Mathematics, Athens University, Athens, Greece

E-mail address: sargyros@math.uoa.gr

Current address: Department of Mathematics, National Technical University of Athens, Athens 15780, Greece

E-mail address: sargyros@math.ntua.gr 